\title{
A Novel Retinal Oscillation Mechanism in an Autosomal Dominant Photoreceptor Degeneration Mouse Model
}

\author{
Hung-Ya Tu ${ }^{1,2,3}$, Yu-Jiun Chen ${ }^{1}$, Adam R. McQuiston ${ }^{4}$, Chuan-Chin Chiao ${ }^{2,3,5}$ and \\ Ching-Kang Chen ${ }^{1,6,7 *}$
}

\begin{abstract}
${ }^{1}$ Department of Ophthalmology, Baylor College of Medicine, Houston, TX, USA, ${ }^{2}$ Institute of Molecular Medicine, National Tsing Hua University, Hsinchu, Taiwan, ${ }^{3}$ Department of Life Science, National Tsing Hua University, Hsinchu, Taiwan,

${ }^{4}$ Department of Anatomy and Neurobiology, Virginia Commonwealth University, Richmond, VA, USA, ${ }^{5}$ Institute of Systems Neuroscience, National Tsing Hua University, Hsinchu, Taiwan, ${ }^{6}$ Department of Biochemistry and Molecular Biology, Baylor College of Medicine, Houston, TX, USA, ${ }^{7}$ Department of Neuroscience, Baylor College of Medicine, Houston, TX, USA
\end{abstract}

\section{OPEN ACCESS}

Edited by:

Steven F. Stasheff,

University of lowa, USA

Reviewed by:

Joshua Singer, University of Maryland, USA

Maureen Ann McCall, University of Louisville, USA

*Correspondence:

Ching-Kang Chen

ching-kang.chen@bcm.edu

Received: 01 September 2015 Accepted: 21 December 2015

Published: 12 January 2016

Citation:

Tu H-Y, Chen Y-J, McQuiston AR, Chiao C-C and Chen C-K (2016)

A Novel Retinal Oscillation

Mechanism in an Autosomal

Dominant Photoreceptor

Degeneration Mouse Model.

Front. Cell. Neurosci. 9:513.

doi: 10.3389/fncel.2015.00513
It has been shown in rd1 and rd10 models of photoreceptor degeneration (PD) that inner retinal neurons display spontaneous and rhythmic activities. Furthermore, the rhythmic activity has been shown to require the gap junction protein connexin 36, which is likely located in All amacrine cells (All-ACs). In the present study, an autosomal dominant PD model called rho $\triangle$ CTA, whose rods overexpress a C-terminally truncated mutant rhodopsin and degenerate with a rate similar to that of $r d 1$, was used to investigate the generality and mechanisms of heightened inner retinal activity following PD. To fluorescently identify cholinergic starburst amacrine cells (SACs), the rho $\triangle$ CTA mouse was introduced into a combined ChAT-IRES-Cre and Ai9 background. In this mouse, we observed excitatory postsynaptic current (EPSC) oscillation and nonrhythmic inhibitory postsynaptic current (IPSC) in both ON- and OFF-SACs. The IPSCs were more noticeable in OFF- than in ON-SACs. Similar to reported retinal ganglion cell (RGC) oscillation in rd1 mice, EPSC oscillation was synaptically driven by glutamate and sensitive to blockade of NaV channels and gap junctions. These data suggest that akin to rd1 mice, All-AC is a prominent oscillator in rho $\triangle$ CTA mice. Surprisingly, OFF-SAC but not ON-SAC EPSC oscillation could readily be enhanced by GABAergic blockade. More importantly, weakening the All-AC gap junction network by activating retinal dopamine receptors abolished oscillations in ON-SACs but not in OFF-SACs. Furthermore, the latter persisted in the presence of flupirtine, an M-type potassium channel activator recently reported to dampen intrinsic All-AC bursting. These data suggest the existence of a novel oscillation mechanism in mice with PD.

Keywords: retina, starburst amacrine cell, All amacrine cells, oscillation mechanism, photoreceptor degeneration 


\section{INTRODUCTION}

Pathological loss of photoreceptors is common in retinal degeneration and a leading cause of blindness. The resultant retinal dysfunction has been characterized in several rodent photoreceptor degeneration (PD) models at varying time points following photoreceptor loss. One consequence of $\mathrm{PD}$ is a spontaneous hyperactivity in inner retinal neurons, which has been observed in retinal ganglion cells (RGCs) of $r d 1$ mice (Stasheff, 2008; Menzler and Zeck, 2011; Poria and Dhingra, 2015), rd10 mice (Goo et al., 2011; Biswas et al., 2014), and Royal College of Surgeons (RCS) rats (Sauvé et al., 2001). Heightened RGC spontaneous activity often manifests itself as a rhythmic oscillation in membrane potential that may lead to synchronous spiking patterns among RGCs of similar functional classes or to phase-shifted discharges among heterotypic RGC populations (Margolis et al., 2014). Although it is known that the RGC oscillations are driven synaptically (reviewed by Margolis and Detwiler, 2011), the precise mechanisms and origin of oscillation are not completely understood (Ye and Goo, 2007; Borowska et al., 2011; Trenholm et al., 2012; Yee et al., 2012; Choi et al., 2014). Nevertheless, it has been proposed that RGC oscillations in the $r d 1$ mouse model arise from intrinsically bursting AII-ACs (Choi et al., 2014) that transmit their synchronous rhythm to depolarizing ON cone bipolar cells (ONCBs) via a well-known gap junction coupling network (Borowska et al., 2011; Trenholm et al., 2012). The rhythmic activation of bipolar cells then produces rhythmic glutamatergic release at their axon terminals and ultimately the rhythmic activation of RGCs. This hypothesis is supported by the observation that inactivation of connexin 36 (Cx36), a key gap junction protein that plays a major role in the AII-AC gap junction network, eliminated the PD-induced hyperactivity in vitro in RGCs and in vivo in superior colliculus of $r d 10$ mice (Ivanova et al., 2015a). Moreover, oscillations mediated by the AII-AC network could also be measured in OFF RGCs either directly through activation of the OFF pathway or indirectly via OFF cone bipolar cells (OFFCBs) through signinverting glycinergic synapses (Poria and Dhingra, 2015).

The function of inner retinal hyperactivity in PD models remains unknown but it has been suggested that hyperactivity may help maintain RGC dendritic architecture following loss of photoreceptors (Mazzoni et al., 2008). Finding ways to retain retinal circuit integrity is desirable, but heightened activity likely reduces the signal-to-noise ratio in RGC outputs (Yee et al., 2012; Barrett et al., 2015). Current data on RGC oscillation in mice with $\mathrm{PD}$, especially those from $r d 1$ mice, suggests that only a subset of RGCs exhibit rhythmic activity (Margolis et al., 2008; Stasheff, 2008; Borowska et al., 2011; Yee et al., 2014). The underlying reason remains unknown but it may result from different excitatory and inhibitory inputs to different RGC subtypes (Yee et al., 2012). Thus, dissecting retinal circuits driving various RGC subtypes with or without PD-induced oscillatory activity may provide unique insights into RGC diversity and assist in the development of novel therapeutic strategies.

Establishing the generality of RGC hyperactivity across PD models is imperative as it remains unclear whether heightened activity occurs in all PD models. Furthermore, it is unclear which remaining retinal neurons, including amacrine and bipolar cells, play a role in rhythm generation. A study by Jones et al. (2003) has systematically compared the progression of neuronal remodeling following photoreceptor loss in retinas of naturally occurring and genetically engineered animal models. Among the models examined, an autosomal dominant PD model originally named S334ter and hereon referred to as rho $\triangle$ CTA, which expresses a rhodopsin mutant with a premature truncation at Ser334 in retinal rods, was found to exhibit fast photoreceptor death and slow retinal remodeling, similar to those seen in rd1 mice (reviewed by Jones and Marc, 2005). This autosomal dominant PD model has distinct advantages in studying the $\mathrm{PD}$-induced inner retinal oscillation over other models because it is genetically more tractable and easier to combine with other genetic backgrounds. As RGC activity is controled by amacrine cells (ACs) through feedback and/or feed-forward inhibition (Masland, 2012), we focused our studies on these retinal interneurons. However, given that there are many AC subtypes in the retina, we began with the genetically tractable starburst amacrine cells (SACs), which are the sole source of acetylcholine in the retina capable of diffusely modulating RGC excitability (Schmidt et al., 1987) and play an essential role in retinal direction-selective circuits (reviewed by Wei and Feller, 2011). To do this we selectively expressed the fluorescent protein tdTomato in SACs in the rho $\triangle \mathrm{CTA}$ background. This allowed us to target SACs for electrical recordings in whole mount retinas. Using this preparation, we found that heightened activity is robust and rhythmic in ON type SACs (ON-SACs) displaced in the ganglion cell layer (GCL). Pharmacological studies then showed that the interaction between AII-AC and ONCB generates oscillation in ON-SACs of rho $\triangle$ CTA retinas, as proposed for the $r d 1$ model. Unexpectedly, we also found that OFF-SACs whose somata reside in the inner nuclear layer (INL) oscillate but appear to be insensitive to pharmacological manipulations that aim to suppress AII-AC gap junction network or intrinsic bursting. Thus, our results strongly suggest the existence of an additional mechanism that contributes to inner retinal oscillation.

\section{MATERIALS AND METHODS}

\section{Animals}

All experiments were conducted in accordance with the rules and regulations of the National Institutes of Health guidelines for research animals, as approved by the institutional animal care and use committee of Baylor College of Medicine. Acquired from the Jackson Laboratory, the Ai9 tdTomato reporter (B6.Cg-Gt(ROSA)26Sor ${ }^{\text {tm } 9(C A G-t d T o m a t o) H z e} / \mathrm{J}$; Madisen et al., 2010) and the ChAT-IRES-Cre knock-in driver (B6;129S6-

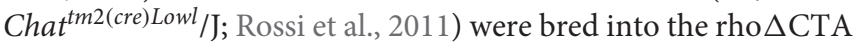
background (Chen et al., 1995). The rho $\Delta$ CTA mouse was a different founder line generated by Jeannie Chen using the same transgenic construct that she used for the making of the published S334ter animals. It was previously used in a retina remodeling study (Jones et al., 2003) and is currently 
maintained in our labs in a mixed 129SvEv and C57BL/6J background. Genotyping was performed by PCR using primers suggested for the reporter and Cre driver as found on-line in the Jackson Laboratory website. The rho $\triangle \mathrm{CTA}$ animals were genotyped using the following primers: RH2: $5^{\prime}$ - TGG GAG ATG ACG ACG CCT AA and RH3: 5' - TGA GGG AGG GGT ACA GAT CC, with a 320 bp product indicating transgene presence. Additional genotyping confirmed the presence of wild type alleles at Pde6b (Pittler and Baehr, 1991) and GPR179 (Balmer et al., 2013) loci. All animals were kept on a $12 / 12 \mathrm{~h}$ light/dark cycle in microisolators with access to food and water ad libitum. For this study, 2-4 month-old male mice with complete degeneration of photoreceptors were used. Animals were deeply anesthetized with isoflurane inhalation, followed immediately by decerebration and harvest of retinas.

\section{Electrophysiology}

Freshly isolated retinas were flat-mounted under room light with the ganglion cell side up and placed onto a punched nitrocellulose membrane. This allows visualization of retinal neurons through differential interference contrast and epifluorescence under an Olympus BX51WI fixed stage microscope (Olympus USA, Central Valley, PA, USA). The retina was superfused $(3-3.5 \mathrm{ml} / \mathrm{min})$ by carbogenated $\left(95 \% \mathrm{O}_{2}\right.$ and $\left.5 \% \mathrm{CO}_{2}\right)$ and temperature-controlled $\left(34-35^{\circ} \mathrm{C}\right.$; Warner instruments $\mathrm{TC}$ $324 \mathrm{~B}$ in-line heater) mammalian Ringer solution (in $\mathrm{mM}$ ): 120 $\mathrm{NaCl}, 5 \mathrm{KCl}, 25 \mathrm{NaHCO}_{3}, 0.8 \mathrm{Na}_{2} \mathrm{HPO}_{4}, 0.1 \mathrm{NaH}_{2} \mathrm{PO}_{4}, 2 \mathrm{CaCl}_{2}$, $1 \mathrm{MgSO}_{4}$ and $10 \mathrm{D}$-glucose. Neurons positive for tdTomato expression in both GCL and INL were targeted for recording using whole cell patch pipettes pulled from borosilicate glass (10-14 M , King Precision Glass, Claremont, CA, USA). For whole cell current clamp recording, a potassium-based internal solution was used (in $\mathrm{mM}$ ): $125 \mathrm{~K}$-gluconate, $8 \mathrm{NaCl}$, 4 ATP-Mg, $0.5 \mathrm{Na}$-GTP, 5 EGTA, 10 HEPES and $0.2 \%$ biocytin $(\mathrm{w} / \mathrm{v})$, and $\mathrm{pH}$ was adjusted to $7.3 \mathrm{using} \mathrm{KOH}$. Cesium-based internal solution containing (in $\mathrm{mM}$ ): 100 Cs-methanesulfonate, $8 \mathrm{NaCl}, 4$ ATP-Mg, $0.5 \mathrm{Na}$-GTP, 5 EGTA, 10 HEPES and $0.2 \%$ biocytin $(\mathrm{w} / \mathrm{v}), \mathrm{pH} 7.3$ adjusted with $\mathrm{CsOH}$, was used for whole cell voltage clamp recording. The liquid junction potentials $(20 \mathrm{mV}$ between Ringer solution and the cesiumbased solution, and $15 \mathrm{mV}$ between Ringer solution and the potassium-based solution) were calculated using Clampex software (Molecular Devices) and corrected after current clamp recording and during voltage clamp recording. Recordings were performed using an AM2400 amplifier (A-M Systems, Sequim, WA, USA) driven by the WinWCP program developed and maintained by John Dempster (University of Strathclyde, Glasgow, UK). Signals were filtered at $5 \mathrm{kHz}$ and sampled at 40 $\mathrm{kHz}$. For current clamp recording, the cells were recorded at rest in free-run mode for 10-15 min without current injection. For voltage clamp recording, cells were held at $-60 \mathrm{mV}$ and repetitively clamped at the reversal potential of chloride $(-75 \mathrm{mV})$ and the reversal potential of glutamate transmission $(0 \mathrm{mV})$. Identities of recorded cells were confirmed post hoc by staining for biocytin and checking for the signature starburst morphology.

\section{Pharmacology}

Drugs were bath-applied via perfusion. Picrotoxin (PTX, $50 \mu \mathrm{M})$ and (1,2,5,6-Tetrahydropyridin-4-yl)methylphosphinic acid (TPMPA, $10 \mu \mathrm{M}$ ) were used simultaneously to inhibit both of $\mathrm{GABA}_{\mathrm{A}}$ and $\mathrm{GABA}_{\mathrm{C}}$ receptors. Strychnine $(1 \mu \mathrm{M})$ was used to block glycinergic transmission. Glutamatergic transmission was blocked by a cocktail of 6-Cyano-7-nitroquinoxaline-2,3dione disodium (CNQX disodium, $10 \mu \mathrm{M}$ ) and D-(-)-2-Amino5-phosphonopentanoic acid (AP5, $10 \mu \mathrm{M}$ ). Tetrodotoxin (TTX, $500 \mathrm{nM}$ ) was used to block voltage-gated sodium channels $(\mathrm{NaV})$. Tubocurarine chloride $(5 \mu \mathrm{M})$ was used to block nicotinic acetylcholine receptors. Meclofenamic acid (MFA, $100 \mu \mathrm{M}$ ) was used as a non-selective gap junction blocker. Dopamine hydrochloride $(100 \mu \mathrm{M})$ was used to reduce gap junction coupling in AII-ACs. Flupirtine maleate $(25 \mu \mathrm{M})$ was used to open the M-type potassium channels. All chemicals were purchased from Sigma-Aldrich or Tocris, prepared fresh and used within $24 \mathrm{~h}$.

\section{Data Analysis}

The power spectral density (PSD) between $0.5-55 \mathrm{~Hz}$ were calculated by fast fourier transform (FFT) of 10 randomly picked $5 \mathrm{~s}$ segments of recordings using OriginPro (Origin Lab, Northampton, MA, USA). Peak oscillation frequency in each PSD profile was verified by visual examination of the raw recording traces. The differences in peak frequency and corresponding power level produced by drug application were analyzed using the Pair-Sample $t$-test, and $p<0.05$ was considered to be significant and marked by asterisks in Figures 3-5.

\section{RESULTS}

To record from both SAC types, we introduced a ChATIRES-Cre driver and a tdTomato reporter into the rho $\triangle$ CTA background. Membrane potentials of both $\mathrm{ON}$ - and OFFSACs were found to oscillate (Figure 1, top), similar to RGC oscillations previously described in $r d 1$ mice. We ensured that the neurons from which we recorded were SACs by performing post hoc morphological analysis. All the recorded neurons displayed signature SAC dendritic morphology and stratification levels in the inner plexiform layer (IPL; Figure 1, bottom).

To investigate the mechanisms of SAC oscillation, we used a cesium-based intracellular solution and voltage clamp recordings to discern between EPSC and IPSC. To isolate EPSC, we clamped the membrane potential of SACs at the calculated reversal potential of chloride $(-75 \mathrm{mV})$. To isolated IPSC, we voltage clamped SAC membrane potentials at the reversal potential for glutamate $(0 \mathrm{mV})$. Using this strategy we found robust rhythmic EPSCs and arrhythmic IPSCs (Figure 2A). Although the proportion of EPSCs observed in ON- and OFF-SACs appeared similar, IPSCs were more frequently detected in OFFSACs. We next quantified membrane current oscillations by performing an FFT analysis to determine the peak frequency of oscillation between $0.5-55 \mathrm{~Hz}$. EPSCs from both ON- and OFF-SACs exhibited a peak frequency near $4 \mathrm{~Hz}$ (Figure 2B). There was no clear frequency peak in PSD of IPSCs. While 


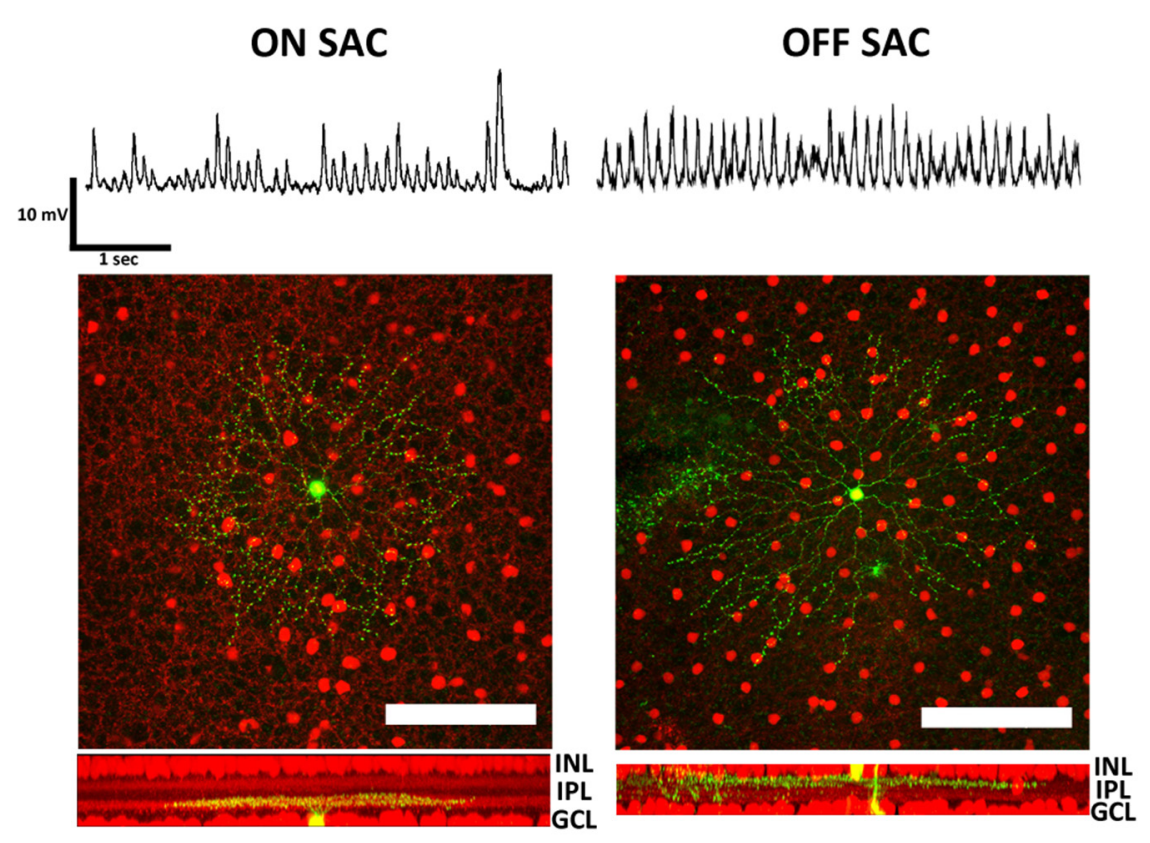

FIGURE 1 | Membrane potential oscillation in retinal ON- and OFF-SACs of the rho $\triangle$ CTA mouse. Membrane potentials in both ON- and OFF-SACs showed robust membrane potential oscillation. Cells (green) targeted for patch clamp recordings in tdTomato expressing retinal cholinergic neurons (red). Cells in both GCL and INL showed typical SAC dendritic morphology and stratification levels in the IPL. GCL, ganglion cell layer; IPL, inner plexiform layer; INL, inner nuclear layer. Scale bar equals $100 \mu \mathrm{m}$.

EPSCs were driven by glutamatergic transmission (see below), IPSCs of the two types of SACs were driven predominantly by GABAergic transmission, as they were attenuated by a mixture of PTX $(50 \mu \mathrm{M})$ and TPMPA $(10 \mu \mathrm{M})$ but not by strychnine $(1 \mu \mathrm{M}$; Figure 2C).

To obtain a more comprehensive picture of how various neurotransmitters may contribute to SAC oscillation, we surveyed the effects of synaptic blockers on rhythmic EPSCs. We discovered that antagonists of ionotropic glutamate receptors (iGluRs; Figures 3A-C) significantly attenuated the oscillation in both SAC types. Furthermore, the polarity of EPSC oscillation in ON-SACs could readily be reversed when cells were held above $0 \mathrm{mV}$ (data not shown), suggesting that iGluRs on ON-SACs are directly involved in oscillation. Thus it is likely that the periodic release of glutamate arises from upstream bipolar cells, including both depolarizing and hyperpolarizing types. Despite being the sole cholinergic neuron in the retina, EPSC oscillations in either SAC type were not affected by an exogenous cholinergic blocker (tubocurarine, $5 \mu \mathrm{M}$; Figures 3G,H). Furthermore, GABAergic inhibition impinging on SACs was not needed for oscillations (Figure 3D) but the frequency of OFF-SAC oscillation could be accelerated by GABAergic blockade (Figures 3E,F). We further noted that GABAergic blockade has no significant effect on $\mathrm{ON}$ SAC oscillation.

The resemblance of SAC oscillation in the rho $\triangle \mathrm{CTA}$ retina to that reported in RGCs of $r d 1$ and $r d 10$ retinas suggested that Cx36-mediated gap junction network among AII-ACs and between AII-ACs and ONCBs (Trenholm et al., 2012) might be involved in rhythmicity. To test this possibility, we used MFA
$(100 \mu \mathrm{M})$ to examine the dependance of SAC oscillation on gap junction coupling (Figure 4A). Rhythmic activity in both ONand OFF-SACs was eliminated by MFA treatment, indicating that gap junction network plays an essential role in the rho $\triangle \mathrm{CTA}$ model. However, MFA is a non-selective and non-specific gap junction blocker and there are many types of connexins in the retina. To seek further support for the involvement of AIIAC gap junction coupling in SAC oscillation, we turned to the reported modulation of $\mathrm{Cx} 36$ coupling in AII-ACs by dopamine. The activation of dopamine D1-like receptor reduced tracer coupling (Kothmann et al., 2009) by dephosphorylating the Ser293 residue of Cx36 in AII-ACs, while other gap junctions may remain permeable when dopamine is present (reviewed by Bloomfield and Völgyi, 2009). Figure 4B shows clearly that EPSC oscillation in ON-SACs (left panels) was disrupted in the presence of exogenous dopamine $(100 \mu \mathrm{M})$, consistent with the speculation that the AII-AC network is involved. To our surprise, however, OFF-SACs remained oscillatory (Figure 4B, right panels), suggesting that another gap junction network insensitive to dopamine sustains OFF-SAC oscillation.

Because electrophysiological support for dopamine-mediated modulation of gap junction conductance between coupled AIIACs is not found (Demb and Singer, 2012; Hartveit and Veruki, 2012), we further explored the different roles that the AIIAC gap junction network plays in the oscillation of ON- vs. OFF-SACs. To do this, we utilized a recently reported intrinsic bursting property of AII-ACs that can be blocked by TTXmediated inhibition of $\mathrm{NaV}$ (Cembrowski et al., 2012) and by flupirtine-mediated activation of M-type potassium currents 

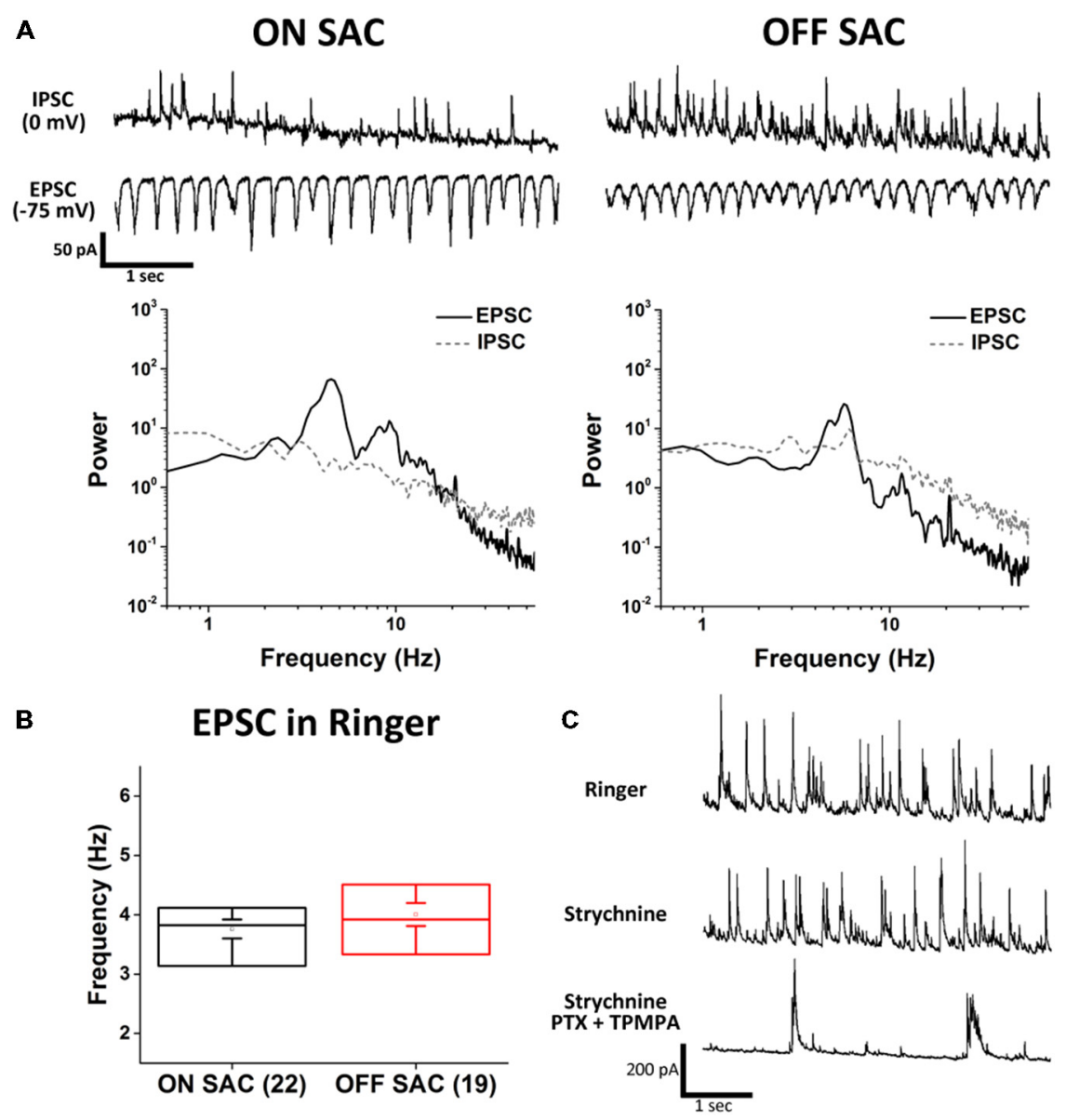

FIGURE 2 | Distinct excitatory and inhibitory inputs underlying membrane potential oscillation in ON- and OFF-SACs. (A) Representative EPSCs and IPSCs from ON- and OFF-SACs with rhythmicity seen only in EPSCs and not in IPSCs. Frequency peaks of oscillation were evident in the power spectral density (PSD) following fast fourier transform (FFT) of recording data. (B) Averaged peak frequencies of EPSC oscillation are similar in ON- and OFF-SACs. Cell numbers are indicated in parentheses. Error bars = SEM. (C) Representative IPSCs seen in an OFF-SAC were inhibited by GABA antagonists but not by glycine receptor antagonist.

(Choi et al., 2014). Application of TTX (500 nM) effectively diminished EPSC oscillation in both SAC types (Figure 5A), although small and irregular activities were still observed in OFF-SACs in the presence of TTX. However, activating M-type potassium channels by flupirtine $(25 \mu \mathrm{M})$ attenuated only EPSC oscillation in ON-SACs and not in OFF-SACs (Figure 5B). Taken together, the differential dopamine and flupirtine effects on the two SAC types strongly support a notion that OFF-SAC oscillation is through a mechanism different from the one driving RGC oscillation in $r d 1$ and ON-SACs in rho $\triangle \mathrm{CTA}$ mice.

\section{DISCUSSION}

The rho $\triangle$ CTA mouse exhibits rapid loss of photoreceptors followed by slow onset of retinal remodeling (reviewed by Jones and Marc, 2005). We found oscillations of SAC membrane potential that require gap junction networks in the retina that are synaptically driven by glutamatergic transmission. The novelty of this investigation lies in the unexpected insensitivity of OFF-SAC oscillation to dopamine and flupirtine that distinguishes it from the mechanism involving the well-characterized AII-AC gap junction network that drives ON-SAC oscillation. Our findings indicate that not one but multiple mechanisms underlie inner retinal hyperactivity following PD. This work warrants a comprehensive survey of RGC diversity and may provide a means to catalog different RGC types according to their connections to distinct retinal circuits in PD animal models. Furthermore, identification of cellular components involved in the novel oscillation mechanism will be helpful in understanding the intricacy of retinal circuitry subserving normal vision.

\section{ON-SAC Oscillations in Wild Type and rho $\triangle$ CTA Mice}

It was reported by Petit-Jacques et al. (2005) that a number of inner retinal neurons, including ON-SACs, displayed rhythmic inward currents in wild type retinas with a frequency similar 


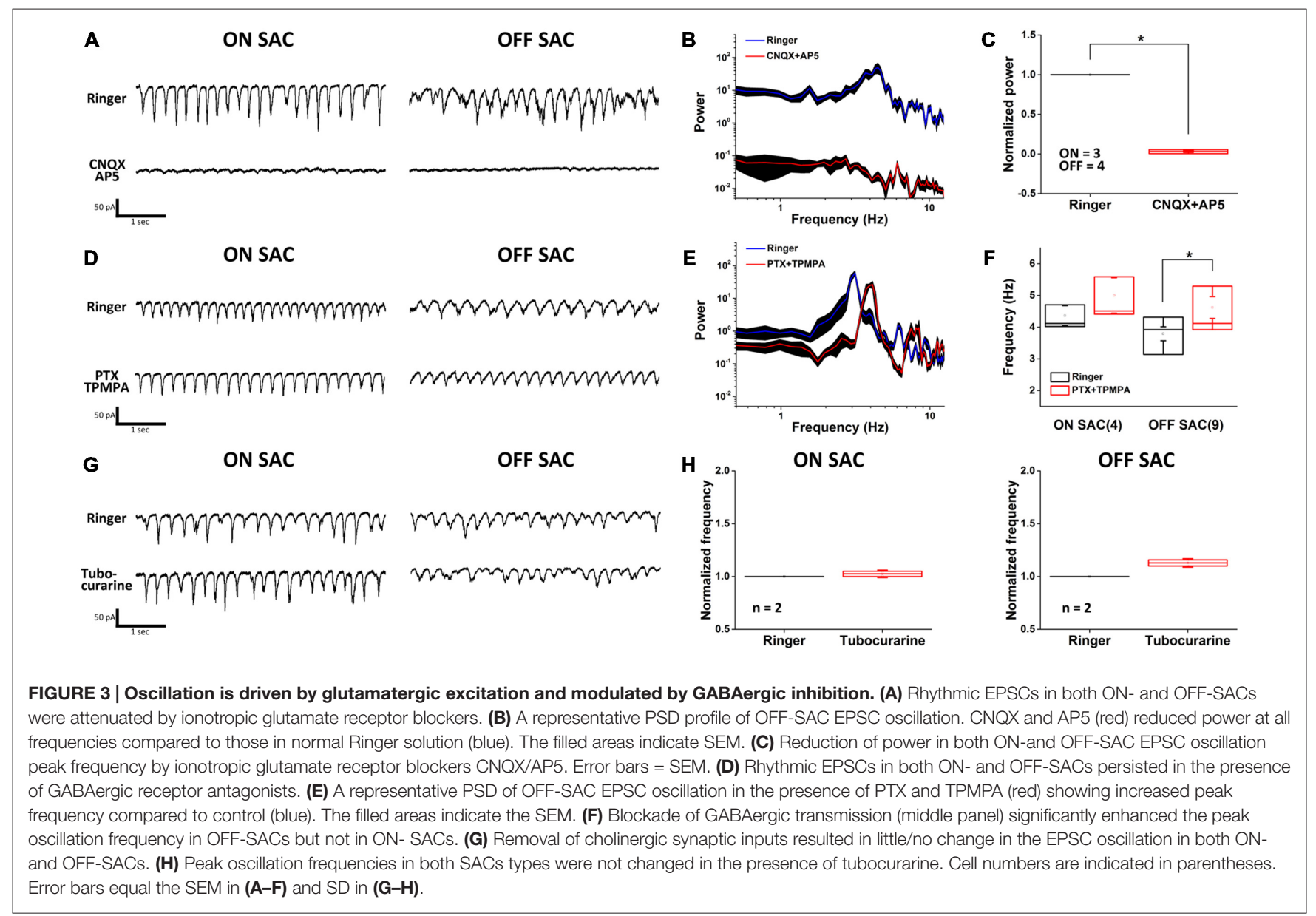

to what we observed in ON-SACs of rho $\triangle \mathrm{CTA}$ retinas. The rhythmic activity in wild type ON-SACs appeared to be driven similarly by synaptically released glutamate. Furthermore, the glutamate release depended on L-type voltage gated calcium channels and could be enhanced by blocking GABAergic transmission or by treating the retina with the non-selective voltage-gated potassium channel blocker Tetraethylammonium chloride (TEA; Petit-Jacques et al., 2005). The same group later reported another light-dependent and faster oscillatory current in wild type ON-SACs with properties similar to spontaneous oscillation (Petit-Jacques and Bloomfield, 2008). The light-dependent oscillation could be blocked by glutamate transporter antagonists or attenuated by GABAergic and glycinergic blockers and by TTX treatment. For the following reasons we consider the oscillation we observed in rho $\triangle \mathrm{CTA}$ ON-SACs different from those of wild type retinas. First, wild type ON-SACs under our recording conditions did not have an obvious peak in PSD, suggesting that if and when they oscillate, it is not as robust as what is routinely seen in mice with PD (data not shown). However, these differences may be explained by the low concentration $(1 \mathrm{mM})$ of TEA purportedly used in their original report to enhance the "small-amplitude" oscillations in approximately half of recorded SACs. Second, blockade of inhibitory transmission enhanced ON-SAC oscillation in wild type retinas but had no effect on oscillation of ON-SACs in rho $\triangle \mathrm{CTA}$ mice. Third, TTX eliminated oscillation in our hands (Figure 5A), but only reduced oscillation amplitude or frequency in theirs (Figure 7 of Petit-Jacques and Bloomfield, 2008). Finally, spontaneous or light-enhanced wild type ON-SAC oscillation could be blocked by inhibiting L-type calcium channels by $30 \mu \mathrm{M}$ Nifedipine, which has no effect on oscillation of ON-SACs in rho $\triangle \mathrm{CTA}$ mice (Chen et. al, unpublished results).

While comparing ON-SAC oscillation between WT and rho $\triangle$ CTA mice may be a fruitful direction to examine the diverse synaptic connections underlying ON-SAC excitability, we shall focus the subsequent discussion solely on rho $\triangle \mathrm{CTA}$ SAC oscillations, whose frequency is noticeably a bit lower than that reported in the $r d 1$ and $r d 10$ mouse RGCs and AII-ACs. An obvious question arises: is this due to a difference between SACs and other inner retinal neurons or a difference between rho $\triangle \mathrm{CTA}$ and other mouse PD models? To answer this question, we have begun to examine oscillation of RGCs in rho $\triangle \mathrm{CTA}$ retinas and found diversity of oscillation characteristics and distinctive sensitivities to pharmacological blockade in different RGC types (Chen et al, unpublished results). Yee et al. (2014) had done a similar but much 


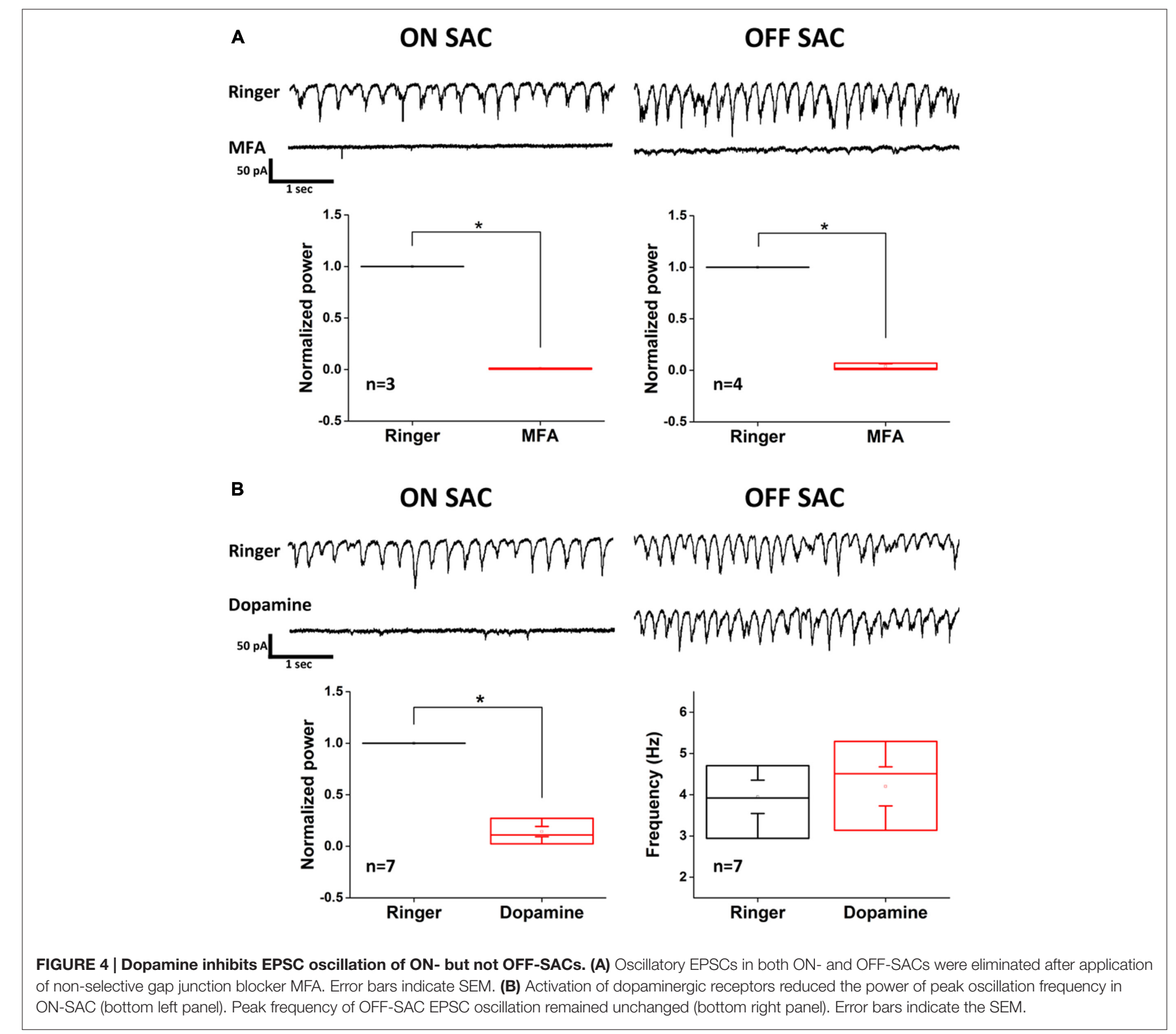

larger survey of oscillating RGCs in $r d 1$ retinas and reported 10 RGC types that oscillated with diverse and distinctive characteristics. Therefore, it appears that SAC oscillation we observed in rho $\triangle \mathrm{CTA}$ mice is not limited to just these two cell types and it is prevalent in many other inner retinal neurons.

\section{Inhibition of ON-SAC Oscillation in rho $\triangle$ CTA Mice by Dopamine and Flupirtine}

The gap junction network of AII-ACs, either the homotypic type amongst themselves or the heterotypic type with ONCBs has been extensively studied. Exogenous dopamine suppresses Cx36 coupling by promoting dephosphorylation at its Ser293 residue (Kothmann et al., 2009). Our data shows that dopamine reversibly blocked ON-SAC oscillation. However, dopamine also triggers diverse actions through its receptors that are abundantly expressed in the retina and is known to modulate ion channel conductance (Pfeiffer-Linn and Lasater, 1996; Witkovsky, 2004; Ichinose and Lukasiewicz, 2007; Popova, 2014). Therefore, our finding that dopamine inhibited the ON-SAC oscillation may not prove the involvement of AII-AC network in SAC oscillation, especially in light of the noted lack of electrophysiological support for modulation of AII-AC gap junction coupling by dopamine (Demb and Singer, 2012; Hartveit and Veruki, 2012). Ivanova et al. (2015b) recently reported morphological and neurochemical aberrations in dopaminergic amacrine cells (DACs) in three mouse PD models and found reduced synaptic contacts between DACs and AII-ACs, raising a possibility that exogenous dopamine merely makes up for weakened modulation of AII-ACs by DACs. To investigate whether AII-ACs indeed play a role, we found that opening the slow and voltage 


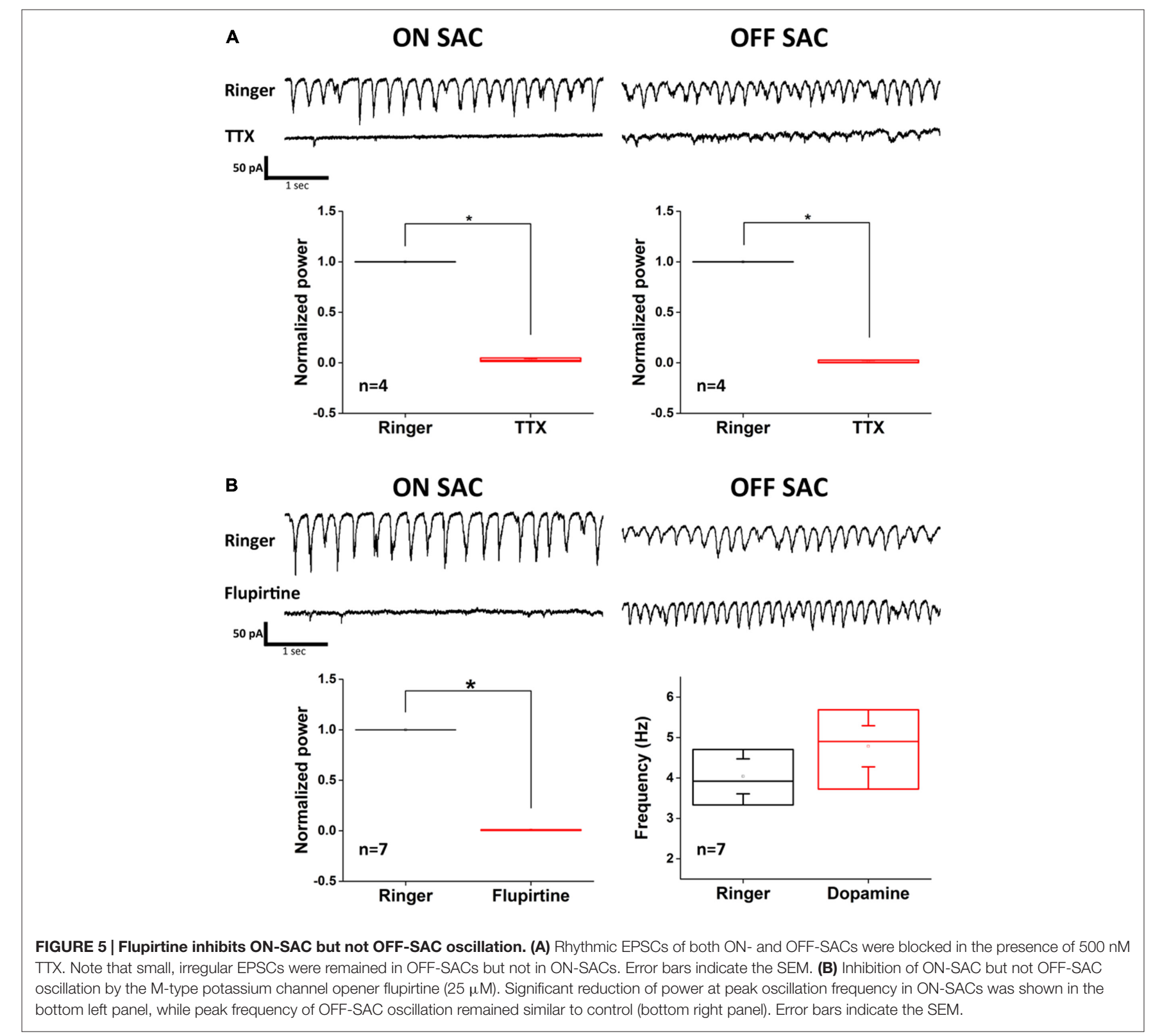

dependent M-type potassium channels by low concentration (25 $\mu \mathrm{M})$ of flupirtine reversibly blocked ON-SAC oscillations in the rho $\triangle$ CTA retinas. Activation of these potassium channels reportedly leads to AII-AC hyperpolarization to a point that its intrinsic bursting becomes inhibited, leading to suppression of RGC oscillation in $r d 1$ retinas (Choi et al., 2014). To further strengthen the notion that the AII-AC network is needed, we predicted and indeed found that low level of TTX (500 nM) robustly prevented ON-SACs from oscillating (Figure 5A). Finally, because MFA effectively blocked oscillation in our hands and a recent report showed the requirement of Cx36 for RGCs to oscillate in $r d 10$ mice (Ivanova et al., 2015a), we have obtained preliminary results from a few morphologically identified ON-SACs in three $\mathrm{C} \times 36^{-/-} /$rho $\triangle \mathrm{CTA}$ mutant mice that $\mathrm{Cx} 36$ is indeed required for ON-SACs in the rho $\triangle \mathrm{CTA}$ background to oscillate (Chen et al., unpublished results). Taken together, these data leave little room to discount the requirement of AII-AC gap junction network in ON-SAC oscillation.

\section{Mechanism Underlying OFF-SAC Oscillation}

The finding in Figures 4B, 5B that OFF-SAC oscillation was insensitive to pharmacological agents capable of blocking ON-SAC oscillation was an exciting observation. Being essential for direction-selective circuits and existing as mirror partners on both sides of the IPL, the mechanism underlying oscillation of OFF-SACs appeared to be different from that of ON-SACs. This finding joins a trending thought that retinal cholinergic 
neurons are not identical (Sun et al., 2013; Ishii and Kaneda, 2014; Borghuis and Fransen, 2015). Regarding the oscillation mechanism, because of its sensitivity to iGluR blockers, MFA, TTX, and more relevantly, insensitivity to strychnine, flupirtine and dopamine, it is reasonable to rule out the direct involvement of the AII-AC network in OFF-SAC oscillation. We speculate here that the periodic glutamate release at hyperpolarizing OFFCB terminals to be the oscillation source. The inhibitory effect of MFA suggests that gap junction coupling among presynaptic OFFCBs may be a possibility. It is known that several types of OFFCBs in mouse retina express connexins (Feigenspan et al., 2004; Hilgen et al., 2011) and at least one type of OFFCBs in rabbit retina has been shown to be gap junction coupled both homo- and heterologously (Mills, 1999). It is therefore of interest to know whether Cx36 is needed for OFF-SACs to oscillate as required in the AII-AC network. Hyperpolarization of AII-ACs resulting from gap junction blockade in $r d 1$ retina (Choi et al., 2014) may reduce the bursting of some types of OFFCBs with $\mathrm{Ca}^{2+}$-dependent regenerative activity (Ma and Pan, 2003). However, the insensitivity of OFF-SAC oscillation to flupirtine suggests that AII-AC hyperpolarization without gap junction uncoupling therein may not be enough to disrupt the OFFCB oscillatory activity. Furthermore, a recent report has identified a restricted set of bipolar cells that express D1 dopamine receptors (Farshi et al., 2015). Since exogenous dopamine has no effect on OFF-SAC oscillation, this suggests that type T1, T3b and T4 OFFCBs may play a negligible role here. We also suspect the involvement of other spiking ACs because of the inhibitory effect of TTX. It is equally possible that OFFCB oscillation is an intrinsic property of the cell, similar to that modeled for AII-ACs, which could be unmasked when outer retina activity is disrupted. While determining the exact oscillation mechanism and cellular components is important, it nonetheless falls beyond the scope of the present study. The notion that more than one oscillation generator exists in retinas following PD suggests that other PD models need to be tested to validate the generality of our findings. Nevertheless, our data indicate for the first time that oscillation of RGCs in PD models such as $r d 1$ and $r d 10$ mice are likely to be more complicated than previously reported and that current knowledge of inner retinal hyperactivity following PD may have seen just a small piece of a larger process.

\section{Oscillation as a Means to Probe Diversity of Inner Retinal Neurons}

Many reports adhere to the idea that inner retinal neurons such as RGCs and ACs are diverse and each subserves vision in unique circuits (Masland, 2011). In published systematic surveys, from conservative to eager estimation, RGCs were clustered into 15-30 types (recently reviewed by Sanes and Masland, 2015) and ACs into around 40 types (MacNeil and Masland, 1998; MacNeil et al., 1999). As all surveys demand meticulous handling of large data sets and depend in varying degrees on morphological features and in some cases genetic markers (Huberman et al., 2009; Rivlin-Etzion et al., 2011; Trenholm et al., 2011), incorporating data on functional connection will conceivably add significantly to such efforts. As the diversity of RGCs in normal and diseased retina remains an active topic of vision research (Völgyi et al., 2009; Pang et al., 2015; Sanes and Masland, 2015), to efficiently harness RGC oscillation in mouse PD models as a classification tool is by all means an onerous task. It demands not only examination of a large number of retinal neurons but also the provision of means or reagents to allow reliable targeting of an identified RGC type with unique oscillation characteristics. Yee et al. (2014) has pioneered a study in this particular direction and defined 10 morphological RGC types in $r d 1$ retinas. With the knowledge that these oscillating neurons may possess differential sensitivity to dopamine or flupirtine, strategies for more in-depth investigation of inner retina oscillation may now be adopted to access neuronal diversity and circuit connectivity. The existence of more than one oscillation mechanisms operating at similar frequencies (Figure 1) also raises an intriguing possibility that an RGC may appear to be non-oscillating (Borowska et al., 2011) but in reality receives out-of-phase inputs from two sources that cancel each other out. Oscillation in this speculated condition can then be unmasked by pharmacological treatment. Using the recording conditions and mouse models depicted here, one may investigate similarity/dissimilarity of hyperactivity among different RGC types with relative ease. Combining the convenience of interrogating circuit connectivity described here with an ever-expanding collection of genetically marked neurons (Sanes and Masland, 2015) will inevitably have an impact on current and future census efforts for RGCs and ACs.

\section{Generality and Function of Neuronal Hyperactivity in Deafferentated Retinas}

This investigation extends our general understanding of inner retinal hyperactivity to include a novel oscillation mechanism, an additional mouse PD model (rho $\triangle \mathrm{CTA}$ ) and to two more AC types (ON- and OFF-SACs). In a different study, we have gathered evidence that this may further be applied to hyperactivity in some mouse models lacking electroretinographic b-waves (Tu et al., 2014, 2015; Chen et al., 2015). One important question emerging from this generalization is whether inner retinal hyperactivity has any biological function, or merely a pathologic fallout following $\mathrm{PD}$ and a nuance to retinal prosthesis and gene/cell therapy fields. Experiments are currently underway to answer these questions.

\section{AUTHOR CONTRIBUTIONS}

Study design: H-YT and C-KC. Experimentation: H-YT, Y-JC, C-KC. Data analysis: H-YT, ARM, C-CC, C-KC. Manuscript preparation: H-YT, ARM, C-CC and C-KC.

\section{FUNDING}

The work is supported by NIH grants EY013811 and EY022228 to C-KC, MH094626 and MH103695 to ARM, an NEI vision core grant EY002520 to the Department of Ophthalmology, Baylor College of Medicine, and an NSC grant NSC-101-2628-B-007001-MY3 to C-CC. 


\section{ACKNOWLEDGMENTS}

We thank Samuel $\mathrm{M}$. Wu for stimulating discussions and Joung Jang, Xin Guan, Daniel Catt, and Viet Chau for excellent technical assistance. The Ophthalmology

\section{REFERENCES}

Balmer, J., Ji, R., Ray, T. A., Selber, F., Gassmann, M., Peachey, N. S., et al. (2013). Presence of the Gpr179(nob5) allele in a C3H-derived transgenic mouse. Mol. Vis. 19, 2615-2625.

Barrett, J. M., Degenaar, P., and Sernagor, E. (2015). Blockade of pathological retinal ganglion cell hyperactivity improves optogenetically evoked light responses in rd1 mice. Front. Cell. Neurosci. 9:330. doi: 10.3389/fncel.2015. 00330

Biswas, S., Haselier, C., Mataruga, A., Thumann, G., Walter, P., and Müller, F. (2014). Pharmacological analysis of intrinsic neuronal oscillations in rd10 retina. PLoS One 9:e99075. doi: 10.1371/journal.pone.0099075

Bloomfield, S. A., and Völgyi, B. (2009). The diverse functional roles and regulation of neuronal gap junctions in the retina. Nat. Rev. Neurosci. 10, 495-506. doi: 10.1038/nrn2636

Borghuis, B. G., and Fransen, J. W. (2015). A temporal gradient of excitatory input contributes to direction selectivity in the dendrites of OFF starburst amacrine cells. Invest. Ophthalmol. Vis. Sci. 56, 2613.

Borowska, J., Trenholm, S., and Awatramani, G. B. (2011). An intrinsic neural oscillator in the degenerating mouse retina. J. Neurosci. 31, 5000-5012. doi: 10. 1523/JNEUROSCI.5800-10.2011

Cembrowski, M. S., Logan, S. M., Tian, M., Jia, L., Li, W., Kath, W. L., et al. (2012). The mechanisms of repetitive spike generation in an axonless retinal interneuron. Cell Rep. 1, 155-166. doi: 10.1016/j.celrep.2011.12.006

Chen, C.-K. J., Bang, A., Chen, Y.-J., McQuiston, A. R., Tu, H.-Y., and Chiao, C.-C. (2015). Cell-autonomous changes in displaced cholinergic amacrine cells lacking Gß5. Invest. Ophthalmol. Vis. Sci. 56, 3231.

Chen, J., Makino, C. L., Peachey, N. S., Baylor, D. A., and Simon, M. I. (1995). Mechanisms of rhodopsin inactivation in vivo as revealed by a $\mathrm{COOH}$ terminal truncation mutant. Science 267, 374-377. doi: 10.1126/science. 7824934

Choi, H., Zhang, L., Cembrowski, M. S., Sabottke, C. F., Markowitz, A. L., Butts, D. A., et al. (2014). Intrinsic bursting of AII amacrine cells underlies oscillations in the rd1 mouse retina. J. Neurophysiol. 112, 1491-1504. doi: 10. 1152/jn.00437.2014

Demb, J. B., and Singer, J. H. (2012). Intrinsic properties and functional circuitry of the AII amacrine cell. Vis. Neurosci. 29, 51-60. doi: 10. $1017 /$ s0952523811000368

Farshi, P., Fyk-Kolodziej, B., Krolewski, D. M., Walker, P. D., and Ichinose, T. (2015). Dopamine D1 receptor expression is bipolar cell type-specific in the mouse retina. J. Comp. Neurol. doi: 10.1002/cne.23932 [Epub ahead of print].

Feigenspan, A., Janssen-Bienhold, U., Hormuzdi, S., Monyer, H., Degen, J., Söhl, G., et al. (2004). Expression of connexin36 in cone pedicles and OFFcone bipolar cells of the mouse retina. J. Neurosci. 24, 3325-3334. doi: 10. 1523/jneurosci.5598-03.2004

Goo, Y. S., Ahn, K. N., Song, Y. J., Ahn, S. H., Han, S. K., Ryu, S. B., et al. (2011). Spontaneous oscillatory rhythm in retinal activities of two retinal degeneration (rd1 and rd10) mice. Korean J. Physiol. Pharmacol. 15, 415-422. doi: 10. 4196/kjpp.2011.15.6.415

Hartveit, E., and Veruki, M. L. (2012). Electrical synapses between AII amacrine cells in the retina: function and modulation. Brain Res. 1487, 160-172. doi: 10. 1016/j.brainres.2012.05.060

Hilgen, G., von Maltzahn, J., Willecke, K., Weiler, R., and Dedek, K. (2011). Subcellular distribution of connexin 45 in OFF bipolar cells of the mouse retina. J. Comp. Neurol. 519, 433-450. doi: 10.1002/cne. 22526

Huberman, A. D., Wei, W., Elstrott, J., Stafford, B. K., Feller, M. B., and Barres, B. A. (2009). Genetic identification of an On-Off direction-selective retinal ganglion cell subtype reveals a layer-specific subcortical map of posterior motion. Neuron 62, 327-334. doi: 10.1016/j.neuron.2009.04.014
Department of Baylor College of Medicine receives a generous unrestricted fund from Research to Prevent Blindness, Inc. C-KC is the Alice R. McPherson Retina Research Foundation Endowed Chair at Baylor College of Medicine.

Ichinose, T., and Lukasiewicz, P. D. (2007). Ambient light regulates sodium channel activity to dynamically control retinal signaling. J. Neurosci. 27, 4756-4764. doi: 10.1523/jneurosci.0183-07.2007

Ishii, T., and Kaneda, M. (2014). ON-pathway-dominant glycinergic regulation of cholinergic amacrine cells in the mouse retina. J. Physiol. Lond. 592, 4235-4245. doi: 10.1113/jphysiol.2014.271148

Ivanova, E., Yee, C. W., Baldoni, R., Jr., and Sagdullaev, B. T. (2015a). Aberrant activity in retinal degeneration impairs central visual processing and relies on Cx36-containing gap junctions. Exp. Eye Res. doi: 10.1016/j.exer.2015.05.013 [Epub ahead of print].

Ivanova, E., Yee, C. W., and Sagdullaev, B. T. (2015b). Disruption in dopaminergic innervation during photoreceptor degeneration. J. Comp. Neurol. doi: 10. $1002 /$ cne.23899 [Epub ahead of print].

Jones, B. W., and Marc, R. E. (2005). Retinal remodeling during retinal degeneration. Exp. Eye Res. 81, 123-137. doi: 10.1016/j.exer.2005.03.006

Jones, B. W., Watt, C. B., Frederick, J. M., Baehr, W., Chen, C. K., Levine, E. M., et al. (2003). Retinal remodeling triggered by photoreceptor degenerations. J. Comp. Neurol. 464, 1-16. doi: 10.1002/cne.10703

Kothmann, W. W., Massey, S. C., and O’Brien, J. (2009). Dopamine-stimulated dephosphorylation of connexin 36 mediates AII amacrine cell uncoupling. J. Neurosci. 29, 14903-14911. doi: 10.1523/JNEUROSCI.3436-09.2009

Ma, Y. P., and Pan, Z. H. (2003). Spontaneous regenerative activity in mammalian retinal bipolar cells: roles of multiple subtypes of voltage-dependent $\mathrm{Ca} 2+$ channels. Vis. Neurosci. 20, 131-139. doi: 10.1017/S0952523803202042

MacNeil, M. A., Heussy, J. K., Dacheux, R. F., Raviola, E., and Masland, R. H. (1999). The shapes and numbers of amacrine cells: matching of photofilled with Golgi-stained cells in the rabbit retina and comparison with other mammalian species. J. Comp. Neurol. 413, 305-326. doi: 10.1002/(sici)10969861(19991018)413:2<305::aid-cne10>3.3.co;2-5

MacNeil, M. A., and Masland, R. H. (1998). Extreme diversity among amacrine cells: implications for function. Neuron 20, 971-982. doi: 10.1016/s0896 6273(00)80478-x

Madisen, L., Zwingman, T. A., Sunkin, S. M., Oh, S. W., Zariwala, H. A., Gu, H., et al. (2010). A robust and high-throughput Cre reporting and characterization system for the whole mouse brain. Nat. Neurosci. 13, 133-140. doi: 10.1038/nn. 2467

Margolis, D. J., and Detwiler, P. B. (2011). Cellular origin of spontaneous ganglion cell spike activity in animal models of retinitis pigmentosa. J. Ophthalmol. 2011:507037. doi: 10.1155/2011/507037

Margolis, D. J., Gartland, A. J., Singer, J. H., and Detwiler, P. B. (2014). Network oscillations drive correlated spiking of ON and OFF ganglion cells in the rd1 mouse model of retinal degeneration. PLoS One 9:e86253. doi: 10.1371/journal. pone. 0086253

Margolis, D. J., Newkirk, G., Euler, T., and Detwiler, P. B. (2008). Functional stability of retinal ganglion cells after degeneration-induced changes in synaptic input. J. Neurosci. 28, 6526-6536. doi: 10.1523/JNEUROSCI.1533-08.2008

Masland, R. H. (2011). Cell populations of the retina: the proctor lecture. Invest. Ophthalmol. Vis. Sci. 52, 4581-4591. doi: 10.1167/iovs.10-7083

Masland, R. H. (2012). The neuronal organization of the retina. Neuron 76, 266-280. doi: 10.1016/j.neuron.2012.10.002

Mazzoni, F., Novelli, E., and Strettoi, E. (2008). Retinal ganglion cells survive and maintain normal dendritic morphology in a mouse model of inherited photoreceptor degeneration. J. Neurosci. 28, 14282-14292. doi: 10. 1523/JNEUROSCI.4968-08.2008

Menzler, J., and Zeck, G. (2011). Network oscillations in rod-degenerated mouse retinas. J. Neurosci. 31, 2280-2291. doi: 10.1523/JNEUROSCI.4238-10.2011

Mills, S. L. (1999). Unusual coupling patterns of a cone bipolar cell in the rabbit retina. Vis. Neurosci. 16, 1029-1035. doi: 10.1017/s0952523899166057

Pang, J. J., Frankfort, B. J., Gross, R. L., and Wu, S. M. (2015). Elevated intraocular pressure decreases response sensitivity of inner retinal neurons in experimental 
glaucoma mice. Proc. Natl. Acad. Sci. U S A 112, 2593-2598. doi: 10.1073/pnas. 1419921112

Petit-Jacques, J., and Bloomfield, S. A. (2008). Synaptic regulation of the light-dependent oscillatory currents in starburst amacrine cells of the mouse retina. J. Neurophysiol. 100, 993-1006. doi: 10.1152/jn.01399. 2007

Petit-Jacques, J., Völgyi, B., Rudy, B., and Bloomfield, S. (2005). Spontaneous oscillatory activity of starburst amacrine cells in the mouse retina. J. Neurophysiol. 94, 1770-1780. doi: 10.1152/jn.002 79.2005

Pfeiffer-Linn, C. L., and Lasater, E. M. (1996). Dopamine modulates unitary conductance of single PL-type calcium channels in Roccus chrysops retinal horizontal cells. J. Physiol. 496(Pt. 3), 607-616. doi: 10.1113/jphysiol.1996. sp021712

Pittler, S. J., and Baehr, W. (1991). Identification of a nonsense mutation in the rod photoreceptor cGMP phosphodiesterase beta-subunit gene of the rd mouse. Proc. Natl. Acad. Sci. U S A 88, 8322-8326. doi: 10.1073/pnas.88.19.8322

Popova, E. (2014). Role of dopamine in distal retina. J. Comp. Physiol. A Neuroethol. Sens. Neural Behav. Physiol. 200, 333-358. doi: 10.1007/s00359014-0906-2

Poria, D., and Dhingra, N. K. (2015). Spontaneous oscillatory activity in rd1 mouse retina is transferred from ON pathway to OFF pathway via glycinergic synapse. J. Neurophysiol. 113, 420-425. doi: 10.1152/jn.00702. 2014

Rivlin-Etzion, M., Zhou, K., Wei, W., Elstrott, J., Nguyen, P. L., Barres, B. A., et al. (2011). Transgenic mice reveal unexpected diversity of on-off directionselective retinal ganglion cell subtypes and brain structures involved in motion processing. J. Neurosci. 31, 8760-8769. doi: 10.1523/JNEUROSCI.0564-11. 2011

Rossi, J., Balthasar, N., Olson, D., Scott, M., Berglund, E., Lee, C. E., et al. (2011). Melanocortin-4 receptors expressed by cholinergic neurons regulate energy balance and glucose homeostasis. Cell Metab. 13, 195-204. doi: 10.1016/j.cmet. 2011.01.010

Sanes, J. R., and Masland, R. H. (2015). The types of retinal ganglion cells: current status and implications for neuronal classification. Annu. Rev. Neurosci. 38, 221-246. doi: 10.1146/annurev-neuro-071714-034120

Sauvé, Y., Girman, S. V., Wang, S., Lawrence, J. M., and Lund, R. D. (2001). Progressive visual sensitivity loss in the royal college of surgeons rat: perimetric study in the superior colliculus. Neuroscience 103, 51-63. doi: 10.1016/s03064522(00)00557-1

Schmidt, M., Humphrey, M. F., and Wassle, H. (1987). Action and localization of acetylcholine in the cat retina. J. Neurophysiol. 58, 997-1015.

Stasheff, S. F. (2008). Emergence of sustained spontaneous hyperactivity and temporary preservation of OFF responses in ganglion cells of the retinal degeneration (rd1) mouse. J. Neurophysiol. 99, 1408-1421. doi: 10.1152/jn. 00144.2007
Sun, L. O., Jiang, Z., Rivlin-Etzion, M., Hand, R., Brady, C. M., Matsuoka, R. L., et al. (2013). On and off retinal circuit assembly by divergent molecular mechanisms. Science 342:1241974. doi: 10.1126/science.1241974

Trenholm, S., Borowska, J., Zhang, J., Hoggarth, A., Johnson, K., Barnes, S., et al. (2012). Intrinsic oscillatory activity arising within the electrically coupled AII amacrine-ON cone bipolar cell network is driven by voltage-gated $\mathrm{Na}+$ channels. J. Physiol. 590, 2501-2517. doi: 10.1113/jphysiol.2011.225060

Trenholm, S., Johnson, K., Li, X., Smith, R. G., and Awatramani, G. B. (2011). Parallel mechanisms encode direction in the retina. Neuron 71, 683-694. doi: 10.1016/j.neuron.2011.06.020

Tu, H.-Y., Bang, A., Mcquiston, A. R., Chiao, C.-C., and Chen, C.-K. J. (2014). Increased dendritic branching in direction selective retinal ganglion cells in nob1 mice. Invest. Ophthalmol. Vis. Sci. 55, 712.

Tu, H.-Y., Chen, Y.-J., Chiao, C.-C., Mcquiston, A. R., and Chen, C.-K. J. (2015). Rhythmic membrane potential fluctuations of cholinergic amacrine cells in mice lacking ERG b-waves. Invest. Ophthalmol. Vis. Sci. 56, 3227.

Völgyi, B., Chheda, S., and Bloomfield, S. A. (2009). Tracer coupling patterns of the ganglion cell subtypes in the mouse retina. J. Comp. Neurol. 512, 664-687. doi: $10.1002 /$ cne. 21912

Wei, W., and Feller, M. B. (2011). Organization and development of directionselective circuits in the retina. Trends Neurosci. 34, 638-645. doi: 10.1016/j.tins. 2011.08.002

Witkovsky, P. (2004). Dopamine and retinal function. Doc. Ophthalmol. 108, 17-40. doi: 10.1023/b:doop.0000019487.88486.0a

Ye, J. H., and Goo, Y. S. (2007). The slow wave component of retinal activity in $\mathrm{rd} / \mathrm{rd}$ mice recorded with a multi-electrode array. Physiol. Meas. 28, 1079-1088. doi: 10.1088/0967-3334/28/9/009

Yee, C. W., Toychiev, A. H., Ivanova, E., and Sagdullaev, B. T. (2014). Aberrant synaptic input to retinal ganglion cells varies with morphology in a mouse model of retinal degeneration. J. Comp. Neurol. 522, 4085-4099. doi: 10. $1002 /$ cne. 23660

Yee, C. W., Toychiev, A. H., and Sagdullaev, B. T. (2012). Network deficiency exacerbates impairment in a mouse model of retinal degeneration. Front. Syst. Neurosci. 6:8. doi: 10.3389/fnsys.2012.00008

Conflict of Interest Statement: The authors declare that the research was conducted in the absence of any commercial or financial relationships that could be construed as a potential conflict of interest.

Copyright (c) $2016 \mathrm{Tu}$, Chen, McQuiston, Chiao and Chen. This is an open-access article distributed under the terms of the Creative Commons Attribution License (CC BY). The use, distribution and reproduction in other forums is permitted, provided the original author(s) or licensor are credited and that the original publication in this journal is cited, in accordance with accepted academic practice. No use, distribution or reproduction is permitted which does not comply with these terms. 